P3 (continued)

Results: The pre-game scores of POMS $2^{\circledR}-Y$ negative items in the baton twirling players were significantly higher than those in hand ball players. On the other hand, the post-game scores showed no significant differences between the two groups. Correlations were found between the scores in most of the negative items of POMS $2^{\circledR}-\mathrm{Y}$ and AEBS total score.

Conclusions and Implications: We suggested that aesthetic athletes had more negative mood states related to disordered eating than non-aesthetic athletes before games. Nutrition education should be conducted with an emphasis on these psychologically characteristics in aesthetic athletes. Funding: None

\section{P4 Associations Between Elementary School Children's Self-Efficacy for Eating Fruits and Vegetables (FV): Observed Fruit and Vegetable Intake and Skin Carotenoid Concentration}

Damon Joyner, BS, Utah State University;

Sheryl Aguilar, $M S, R D$, Utah State University;

Heidi Wengreen, $P h D, R D$, heidi.wengreen@usu.edu, Utah State University, NDFS Department 8700 Old Main Hill, Logan, Utah 84322

Objective: To evaluate associations between elementary school children's self-efficacy for eating FV, observed lunch-time FV intake, and skin carotenoid concentrations. Study Design: Children $(n=603)$ in two elementary schools in grades 1-6 were invited to participate. Self-efficacy for eating FV was assessed using a six-item interviewer-assisted questionnaire. Lunch-time FV intake was assessed over three days using digital observation. Skin carotenoid concentrations were measured using a portable Raman spectroscopy device. Pearson correlation coefficients and linear regression were used to examine associations of interest.

Results: Average lunch-time fruit intake, vegetable intake, and skin carotenoid concentration were 13 grams (+/- 20 grams), 33 grams (+/- 31 grams) and 30607 units (+/- 14026 units), respectively. Vegetable intake and skin carotenoid concentration were significantly correlated with self efficacy (vegetable $r=0.23, p<0.0001$; skin carotenoid $\mathrm{r}=0.22, \mathrm{p}<0.0001)$ but fruit consumption was not $(\mathrm{r}=.04, \mathrm{p}=0.49)$. The range of self-efficacy scores was 6-18, with higher scores representing higher self-efficacy. A linear regression model controlling for weight, gender, and grade predicted that for every unit increase of self efficacy, skin carotenoids increased by 1011 units $(\mathrm{p}=0.004)$.

Conclusion: Self-reported self-efficacy for eating FV is associated with higher vegetable intake and skin carotenoid concentrations in elementary school aged children. As items in the self-efficacy questionnaire asked about children's confidence in their ability to eat FV, it is unclear if self efficacy drives healthy eating or if healthy eating drives self efficacy. Future studies should experimentally increase children's dietary self-efficacy and evaluate the effects of this manipulation on FV consumption.

Funding: $\mathrm{NIH}$

\section{P5 Broader Implications of Nutrition} Education on High School Success Markers

Katera Moore, PhD, MES, kymoore@sas.upenn.edu, University of Pennsylvania, 3451 Walnut Street, Suite P117A, Philadelphia, PA 19104; Melissa Sattler, MSPH, RD, University of Pennsylvania

Objective: To explore the impact of out-of-school-time nutrition education on markers of academic success by considering students' attendance, days tardy, and GPA.

Study Design, Setting, Participants, Intervention: As part of the Growing Together Youth Empowerment Project (YEP), students from William L. Sayre and Paul Robeson High Schools participated in group nutrition education 15 hours per week in the after-school setting with a community service component. Both of these School District of Philadelphia schools are in communities that experience high rates of poverty, violence, and chronic disease.

Outcome Measures and Analysis: GPA, days absent, and days tardy between September 7, 2016 and February 6, 2017 were analyzed and averaged using report cards of 23 students from Sayre and 18 students from Robeson. Data for the student body was provided by administrative staff at each school. The results reflect the different methods used by schools to collect data.

Results: On average, YEP Sayre students were present 86\% of days and tardy $23.3 \%$; this compares to Sayre's average attendance rates of $53 \%$ of students present and $23.2 \%$ tardy. YEP Robeson students missed an average of 6.1 days and were tardy 3.8 days compared to 7.05 days absent and 2.87 days tardy. GPA comparison data was only available at Robeson with YEP students averaging 2.4 and all students averaging 2.35 .

Conclusions and Implications: Nutrition education is often ancillary to high school curriculum. This study demonstrates that nutrition education can have broader impacts when compared to students not in the program, particularly around the development of 21st Century Skills. This has implications for developing programs aimed at improving outcomes for high school students in under-resourced urban areas.

Funding: Supplemental Nutrition Assistance Program Education, Office of Minority Health

\section{P6 Classroom Characteristics and Their Association with Nutrition Education Program Outcomes: A Multi-level Analysis of FoodMASTER Intermediate}

Kathryn I. Hoy,LDN, RD, MFN, khoy@email.sc.edu, University of South Carolina, HPEB, Discovery Building, 915 Greene Street, Columbia, SC 29208;

Caroline Dunn, MS, LDN, RD, University of South Carolina; Virginia C. Stage, $P h D, L D N, R D$, East Carolina University; Melani W. Duffin, PhD, LDN, RD

Objective: To examine the relationship between student and classroom-level characteristics on nutrition knowledge after completion of the FoodMASTER Intermediate

Continued on page $S 25$ 
P6 (continued)

(FMI) integrated nutrition education program among 4 th grade students, as compared to a similar group of control students.

Study Design, Settings, Participants, Intervention: The sample consisted of $n=263$ students nested in 18 classrooms in North Carolina and Ohio. Nine classrooms used FMI and nine used traditional mathematics and science curriculum in a quasi-experimental design.

Outcome Measures and Analysis: Multi-level models were used to assess the relationship between student-level variables including: gender, free and reduced lunch status (as a proxy for student socioeconomic status), and baseline nutrition knowledge; classroom-level variables including: control or intervention status, teacher experience and teacher nutrition training on student nutrition knowledge at the conclusion of the program. A total of five models were built and analyzed using PROC MIXED and estimated using maximum likelihood in SASv9.4.

Conclusions and Implications: Classroom-level variables including curriculum and teacher characteristics accounted for $\sim 56 \%$ of variability in nutrition knowledge after the program. The FoodMASTER intervention was found to be a significant predictor of nutrition knowledge. Students exposed to FMI score on average 4.32 points higher than control classrooms $(\mathrm{p}=.0001)$. Teachers with $8+$ years of experience were found to improve predicted post-nutrition knowledge scores $(\mathrm{p}=.006)$. Based on these analyses, integrated nutrition education such as FoodMASTER significantly increases student nutrition knowledge. Further, there is a moderating influence of teacher experience indicating that effectiveness of nutrition education programs may be improved when administered by experienced teachers.

Funding: None

\section{P7 Clustering of Youth Empowerment and Health Behaviors Among Sixth to Eighth Grade Adolescents}

Biyi Chen, MS, South Dakota State University; Kendra Kattelmann, PhD, RDN, LN, FAND, kendra. kattelmann@sdstate.edu, South Dakota State University, Box 2203, Health and Nutritional Sciences Department, Brookings, SD 57006; Ann Michelle Daniels, PhD, South Dakota State University; Koushik Adhikari, PhD, University of Georgia; Susan Zies, MEd, Ohio State University Extension; Erika Lindshield, MPH, RDN, $L D$, Kansas State University; Tandalayo Kidd, $P h D, R D, L P N$

Objective: To identify clusters of perceived youth empowerment among sixth to eighth grade adolescents and their cross-sectional associations with demographic indicators; to examine the distribution of health behaviors (including nutrition and physical activity (PA) behaviors), as identified clusters, among genders.

Study Design, Settings, Participants, Intervention: This baseline study was conducted as part of a five-year tri-state community-based participatory research (CBPR) project-Ignite: Sparking Youth to Create Healthy Commu- nities. A total of 282 six to eighth grade adolescents from South Dakota, Kansas and Ohio completed questionnaires related to youth health behaviors and perceived youth empowerment.

Outcome Measures and Analysis: The cluster analysis identified groups of adolescents with similar perceived youth empowerment. Chi-square tests assessed cluster differences by gender, ethnicity, and urban/rural status. Analyses were conducted using SAS 9.4 software.

Results: Two clusters were identified empowered youth (EY) $(n=90)$ and "un-empowered youth (UY)" $(\mathrm{n}=192)$. For both genders, the "EY" cluster had significantly greater ( $\mathrm{P}$ $<0.05$ ) health promoting behaviors which included greater self-efficacy, motivation, perceived social support in regards to healthy behaviors, positive health beliefs and less perceived barriers to healthy eating and PA when compared to UY. In addition, the youth in the EY cluster reported more time for PA (total at least 60 minutes/day) per week.

Conclusions and Implications: Promoting youth empowerment may be an effective way of informing interventions designed to foster engagement in health-promoting behaviors among adolescents. Further research should investigate the influence for youth empowerment to promote the health development in youth and create greater community change.

Funding: NIFA

\section{P8 Cognitive Interviews Define Fuel for Fun Physical Activity Survey Items as Face Valid with Rochester, NY Area Fourth Graders}

Elizabeth Wayman, MS, Rochester Institute of Technology; Leslie Cunningham-Sabo, PhD, RD, Colorado State University; Barbara Lohse, PhD, RD, CDN, balihst@rit.edu, Rochester Institute of Technology, 180 Lomb Memorial Drive, CIMS 78-A622, Rochester, NY 14623

Objective: Assess face validity of tested physical activity (PA) self-report items used to evaluate Fuel for Fun with a new 4th grade audience.

Study Design, Setting, Participants: Face-to-face, audio-recorded cognitive interviews conducted at library or school venues with a convenience sample of children from the Rochester, NY area.

Outcome Measures and Analysis: Interview transcripts were analyzed with Atlas.ti using a content analysis approach. Coherence and congruence with reference definitions were assessed for descriptions or amount of strenuous, moderate, mild, sedentary activity and practices/intentions toward PA to denote stage of change for PA behavior.

Results: Students ( $\mathrm{n}=24 ; 50 \%$ girls; $92 \%$ white; $12 \%$ Hispanic) ranged from 8 to $10 \mathrm{y}(\mathrm{M}=9.70 \pm .43 \mathrm{y})$ and attended nine schools (three urban, six suburban). Four students attended schools with free/reduced lunch participation $\geq 50 \%$ of the student body. Mean interview length was $29 \pm 4 \mathrm{~min}$. Stage of change was action/maintenance for $87 \%$, with $172 \pm 110 \mathrm{~min} /$ week strenuous activity, $155 \pm 122 \mathrm{~min} /$ week moderate, $124 \pm 136 \mathrm{~min} /$ week mild, and $2.5 \pm 2.2$ hours/day sedentary activity. Although only $17 \%$ demonstrated comprehension of the terms strenuous,

Continued on page S26 